\title{
Estimating 3D L5/S1 moments and ground reaction forces during trunk bending using a full-body ambulatory inertial motion capture system
}

\author{
G.S. Faber ${ }^{\text {a,b,c,*}, ~ C . C . ~ C h a n g ~}{ }^{\text {c,d }}$, I. Kingma ${ }^{a}$, J.T. Dennerlein ${ }^{\text {b,e }}$, J.H. van Dieën ${ }^{a}$ \\ ${ }^{a}$ Department of Human Movement Sciences, Faculty of Behaviour and Movement Sciences, Vrije Universiteit Amsterdam, \\ MOVE Research Institute Amsterdam, The Netherlands \\ ${ }^{\mathrm{b}}$ Department of Environmental Health, Harvard T.H. Chan School of Public Health, Boston, MA, USA \\ ${ }^{\mathrm{c}}$ Liberty Mutual Research Institute for Safety, Hopkinton, MA, USA \\ d Department of Industrial Engineering \& Engineering Management National Tsing Hua University, Taiwan, ROC \\ e Department of Physical Therapy, Movement, and Rehabilitation Sciences, Northeastern University, Boston, MA, USA
}

\section{A R T I C L E I N F O}

Article history:

Accepted 20 November 2015

\section{Keywords:}

Inertial measurement unit (IMU)

Inertial sensors

Low back loading

Physical exposure

Occupational biomechanics

\begin{abstract}
A B S T R A C T
Inertial motion capture (IMC) systems have become increasingly popular for ambulatory movement analysis. However, few studies have attempted to use these measurement techniques to estimate kinetic variables, such as joint moments and ground reaction forces (GRFs).

Therefore, we investigated the performance of a full-body ambulatory IMC system in estimating 3D L5/S1 moments and GRFs during symmetric, asymmetric and fast trunk bending, performed by nine male participants. Using an ambulatory IMC system (Xsens/MVN), L5/S1 moments were estimated based on the upper-body segment kinematics using a top-down inverse dynamics analysis, and GRFs were estimated based on full-body segment accelerations.

As a reference, a laboratory measurement system was utilized: GRFs were measured with Kistler force plates (FPs), and L5/S1 moments were calculated using a bottom-up inverse dynamics model based on FP data and lower-body kinematics measured with an optical motion capture system (OMC). Correspondence between the OMC + FP and IMC systems was quantified by calculating root-mean-square errors (RMSerrors) of moment/force time series and the interclass correlation (ICC) of the absolute peak moments/forces.

Averaged over subjects, L5/S1 moment RMSerrors remained below $10 \mathrm{Nm}$ (about $5 \%$ of the peak extension moment) and 3D GRF RMSerrors remained below $20 \mathrm{~N}$ (about $2 \%$ of the peak vertical force). ICCs were high for the peak L5/S1 extension moment (0.971) and vertical GRF (0.998). Due to lower amplitudes, smaller ICCs were found for the peak asymmetric L5/S1 moments (0.690-0.781) and horizontal GRFs (0.559-0.948).

In conclusion, close correspondence was found between the ambulatory IMC-based and laboratory-based estimates of back load.
\end{abstract}

(c) 2015 Elsevier Ltd. All rights reserved.

\section{Introduction}

Mechanical loading of the spine has been identified as an important risk factor for the development of low back pain (Coenen et al., 2013; da Costa and Vieira, 2010; Kuiper et al., 2005), which still is a major global medical/economic burden (Vos et al., 2012). Therefore, many studies have investigated the effect of ergonomic interventions on mechanical back loading, usually employing laboratory equipment such as force plates (FPs) and optical motion capture systems (OMC).

\footnotetext{
* Corresponding author at: Department of Human Movement Sciences, Faculty of Behaviour and Movement Sciences, Vrije Universiteit Amsterdam, MOVE Research Institute Amsterdam, The Netherlands. Tel.: +31 2059 88566; fax: +31 205988529.

E-mail address: gertfaber.sci@gmail.com (G.S. Faber).
}

Although valuable information can be obtained from these laboratory measurements, there is a growing need for low back load assessment outside the laboratory, e.g., epidemiological surveys (Kuiper et al., 1999) and intervention studies (Lötters and Burdof, 2002). For such field studies it is often infeasible to utilize the above mentioned laboratory measurement techniques. Therefore, earlier biomechanical field studies used video analysis (Coenen et al., 2013; Norman et al., 1998). However, such studies are rare, probably because the laborious frame-by-frame data analysis. To overcome this limitation, recently wearable measurement systems have been developed for ambulatory assessment of back loading (Freitag et al., 2007; Marras et al., 2010). However, these new measurement systems are bulky. An alternative wearable system, which is less bulky and can even be worn under the clothes, is and inertial/magnetic motion capture (IMC) system, consisting of small inertial sensors (ISs) measuring 3D 
segment motions. Many studies have shown the validity of the IMC system for measurement of kinematics (Cutti et al., 2008; Faber et al., 2013b; Godwin et al., 2009; Luinge and Veltink, 2005; Plamondon et al., 2007; Roetenberg et al., 2005). However, the number of studies testing the validity of the system for the assessment of the kinetics (e.g. joint moments) is limited (Godwin, 2009; Kim and Nussbaum, 2013; van den Noort et al., 2008).

Therefore, we studied the performance of a full-body IMC system for estimating 3D L5/S1 moments during trunk bending. As a reference, L5/S1 moments were calculated using a state-of-theart laboratory measurements system, measuring GRFs with FPs and measuring full-body kinematics with an OMC system. In addition to L5/S1 moments, we also evaluated how well ground reaction forces (GRF) due to body accelerations can be estimated. The latter could potentially be used to estimate hand forces (Faber et al., 2013a) when combined with ambulatory GRFs measurements (Faber et al., 2009b). Moreover, since GRF can be measured directly and accurately this provides an accurate tool to validate IMC-based kinetic analyses.

\section{Methods}

\subsection{Subjects and experimental procedures}

Nine healthy male subjects (age: $36 \pm 11$ years, mass: $81 \pm 12 \mathrm{~kg}$, height: $178 \pm 9 \mathrm{~cm}$ ) participated in the experiment that was approved by institutional review boards of the Harvard T.H. Chan School of Public Health and the Liberty Mutual Research Institute for Safety. After providing written consent, subjects were equipped with all the measurement instrumentation and after some calibration measurements (see following sections) subjects performed the following trunk bending tasks in one trial.

\section{Symmetric trunk bending at normal speed \\ 2. Asymmetric trunk bending at normal speed \\ 3. Symmetric trunk bending at fast speed}

Fast trunk bending was not performed asymmetrically, because of risk of injury. Subjects were verbally instructed about the trunk bending direction ("straight forward" and "about $45^{\circ}$ to the left") and bending speed ('normal', 'fast').

Because of the strongly distorted magnetic field close to the force plates, trials started with the subject standing on a platform next to the measurement volume. After starting the measurement, subjects walked onto force platforms and performed each of the 3 trunk bending tasks once. Total time of the trial was usually below $1 \mathrm{~min}$.

\subsection{Instrumentation and data pre-processing}

GRF were measured with two Kistler FPs, recorded at 200 samples/s (type 9286AA, Kistler Instrumente AG, Winterthur, Switzerland). Furthermore, full-body kinematics were measured with an Xsens IMC system at 120 samples/s (MVN, Xsens technologies B.V., Enschede, the Netherlands) and with an Certus Optotrak OMC system at 50 samples/s (Northern Digital, Waterloo ON, Canada). All signals were resampled to 120 samples/s using linear interpolation.Forces and kinematics were bi-directionally low-pass filtered with a second-order Butterworth filter at $15 \mathrm{~Hz}$ and $5 \mathrm{~Hz}$, respectively. FP and OMC data were measured synchronously and the IMC data was synchronized based on the IMC and OMC resultant angular velocity of the head segment.

For the IMC system, the standard full-body MVN setup was used (Kim and Nussbaum, 2013; Roetenberg et al., 2013) consisting of 17 miniature inertial sensors (ISs). Data was recorded using Xsens software (MVN Studio 3.0, Xsens technologies B.V., Enschede), providing a built-in anatomical human body model. For the OMC system, marker clusters were used to capture segment motion.

For both the OMC and IMC systems, motion sensors (ISs and marker clusters) were attached to the pelvis, head, the upper arms, forearms, thighs, shanks, and feet. In addition, in accordance with the requirements of the built-in anatomical model, ISs were placed on both scapulae, the sternum and hands; and a marker clusters was placed on the posterior side of the thorax. Because most marker clusters were (rigidly) attached to the inertial sensors, only non-magnetic material was used in the clusters (verified with magnetic field IS output).

\section{3. $F P+O M C$ based L5/S1 moments and GRF assessment}

First, FP and OMC data were expressed in the same global coordinate system (Fig. 1). Summing the GRFs measured by the two FPs provided the total GRF. For all 16 OMC modeled body segments (feet, shanks, thighs, pelvis, abdomen, thorax, head, upper arms, forearms and hands), anatomical coordinate systems, center of mass ( $\mathrm{CoM})$ position, and inertial parameters were calculated based on anatomical landmarks that were related to the corresponding marker clusters using a probe with four markers (Cappozzo et al., 1995). L5/S1 moments were calculated based on the GRFs and lower-body kinematics $\left(\mathrm{OMC}_{\text {lower }}\right)$, using a bottom-up inverse dynamics model (Kingma et al., 1996) with improved anthropometric modeling (Faber et al., 2009 a). All data (pre-) processing described above and below was programmed in Matlab.

\subsection{IMC based L5/S1 moments estimation}

For anatomical calibration (relating the ISs to the corresponding segment coordinate systems) of the built-in MVN body-model, stature and segment lengths were inputted into the MVN software and an upright calibration posture was recorded (N-pose) (Roetenberg et al., 2013). The MVN software reports calibration

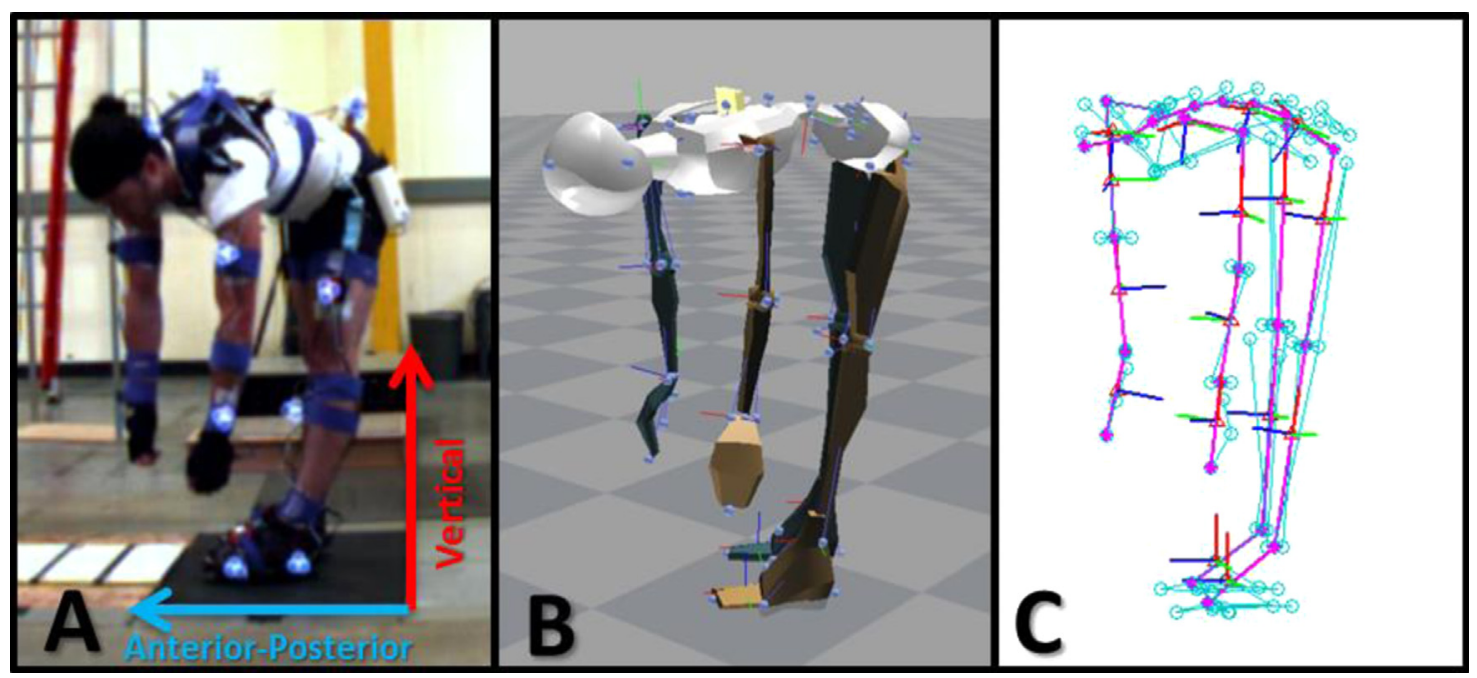

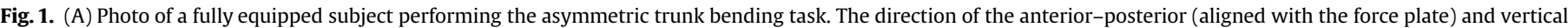

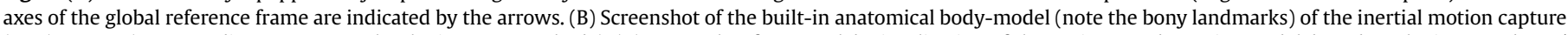

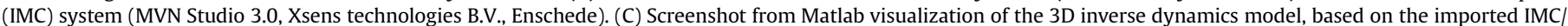
MVN data (note the center of mass of each segment: red triangles). 
quality in one of four levels (good, acceptable, fair and poor), and a minimum of acceptable quality was ensured. The Kinematic Coupling $\left(\mathrm{KiC}^{\mathrm{TM}}\right)$ algorithm was enabled in the software, compensating for possible magnetic disturbances of the lower-body kinematics.

The MVN defines the forward axis of the IMC global coordinate system as the direction of the local earth magnetic field. To align it with the OMC global coordinate system, data were rotated around the common vertical axis, such that the new forward global IMC axis was aligned with the anterior directed pelvis axis measured in the upright position before the first trunk bending.

To estimate full-body segment CoM positions and inertial properties, bony landmark and joint position estimates (including the L5/S1 joint) provided by the built-in MVN body-model were used as input to our 3D model that we also used for the OMC system (same 16 body segments).

MVN provides, based on the IS inertial recordings, for each segment the angular velocity $\left(\boldsymbol{\omega}^{2}\right)$, angular acceleration ( $\left.\boldsymbol{\alpha}\right)$ and the linear acceleration of the origin (a_origin) of the segment (usually the proximal joint) in the earthbound coordinate system. To calculate the segment CoM accelerations (a_CoM) the following equation was used for each segment:

\section{a_CoM $=$ a_origin $+\alpha \times($ CoM - origin $)-\omega^{2}($ CoM - origin $)$}

IMC L5/S1 moments were estimated based on the upper-body segments (IMC upper $_{\text {) }}$ using a "top-down" inverse dynamics model (Kingma et al., 1996). Note that for this analysis, the above calculated segment angular velocities and linear accelerations were used (rather than recalculated based on the MVN position data).

\subsection{IMC based GRF estimation}

IMC GRF was estimated $\left(\mathbf{G R F}_{\text {est }}\right)$ based on above calculated CoM accelerations of all segments $(i)$

$\mathbf{G R F}_{\text {est }}=-\sum_{i=1}^{q}\left[m_{i} *\left(\mathbf{a C o M} \mathbf{M}_{i}-\mathbf{g}\right)\right]$

where $\mathbf{g}$ is the gravitational vector and $q$ is the total number (16) of body segments of the entire full-body model $\left(\mathrm{IMC}_{\mathrm{full}}\right)$.

\subsection{Additional OMC based top-down L5/S1 moments and GRF calculation}

To test if IS measurement error played an important role, the IMC GRF full estimation method described above, was utilized again but now based on the fullbody $\mathrm{OMC}$ kinematics $\left(\mathrm{OMC}_{\text {full }}\right)$. In addition, because reference bottom-up calculated L5/S1 moments are not directly measured like the GRFs but estimated using a 3D inverse dynamics model, which includes a number of assumptions (eg CoM position, mass distribution, rigid segments), it has a limited accuracy. The accuracy

$3 \mathrm{D}$ ground reaction force $(\mathrm{N})$
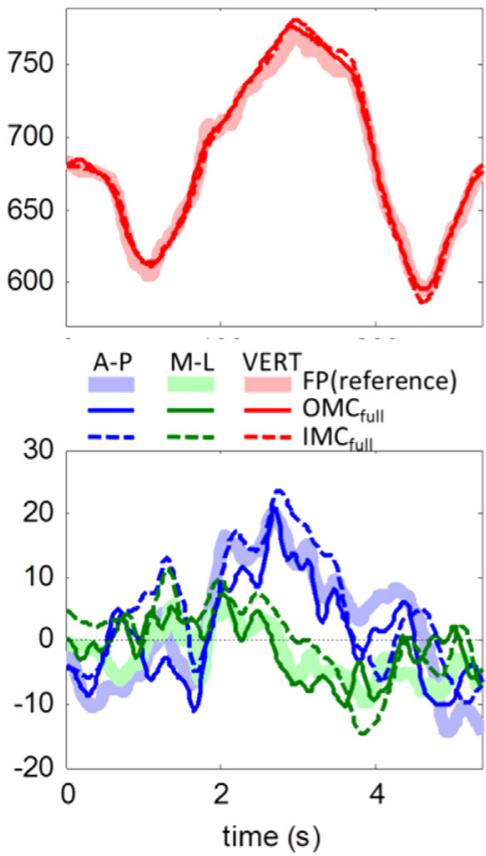

of such inverse dynamics analysis of L5/S1 moments is often quantified by performing a top-down versus bottom-up comparison (Kingma et al., 1996; Plamondon et al., 1996). To verify the validity of the reference OMC based bottom up L5/S1 moment calculations in the current experiment, we also calculated the top-down $\mathrm{L} 5 / \mathrm{S} 1$ moments based on the upper-body $\mathrm{OMC}$ data $\left(\mathrm{OMC}_{\mathrm{upper}}\right)$, identical to the way it was done based on the IMC data.

\subsection{Data reduction and statistics}

The correspondence between the outcomes of the reference OMC+FP system and the IMC system was quantified for all 3D components of the L5/S1 moment (extension, lateral flexion, twist) and of the GRF (vertical, posterior-anterior, mediolateral), for each task separately. For all time series, root-mean-squared errors (RMSerrors) and coefficients of determination $\left(R^{2}\right)$ were calculated. Furthermore, absolute peak values extracted from the time series of the reference OMC + FP system were compared to absolute peaks of the IMC system using paired $t$-tests. Finally, for the absolute peak values of all the tasks together, intraclass correlations coefficients (ICCs) were calculated using ICC(2,1) (Shrout and Fleiss, 1979).

The analysis described above was repeated, now comparing the reference $\mathrm{FP}+\mathrm{OMC}$ system outcomes (L5/S1 moments and GRF) with the additional $\mathrm{OMC}_{\text {upper }}$ based top-down L5/S1 moments and with the additional $\mathrm{OMC}_{\text {full }}$ based GRF estimation. Furthermore, the correspondence (RMSerrors, $R^{2}$ and ICCs) was statistically compared to correspondence between the OMC+FP system and the IMC system described above. For the RMSerrors and $R^{2}$-values this was done using paired $t$ tests. ICCs were considered significantly different in case the confidence intervals did not overlap.

\section{Results}

Fig. 2 presents a typical example of an asymmetric trunk bending trial, showing the GRF and 3D L5/S1 moment time series calculated based on the reference laboratory system analyses (FP \& $\mathrm{FP}+\mathrm{OMC}_{\text {lower }}$ ), the IMC system analyses $\left(\mathrm{IMC}_{\text {full }} \& \mathrm{IMC}_{\mathrm{upper}}\right)$, and the additional laboratory system analyses $\left(\mathrm{OMC}_{\text {full }} \& \mathrm{OMC}_{\text {upper }}\right)$.

\subsection{L5/S1 Moments}

\subsubsection{Agreement of L5/S1 moment time series}

For symmetric and asymmetric as well as fast trunk bending, the correspondence between the L5/S1 extension moment

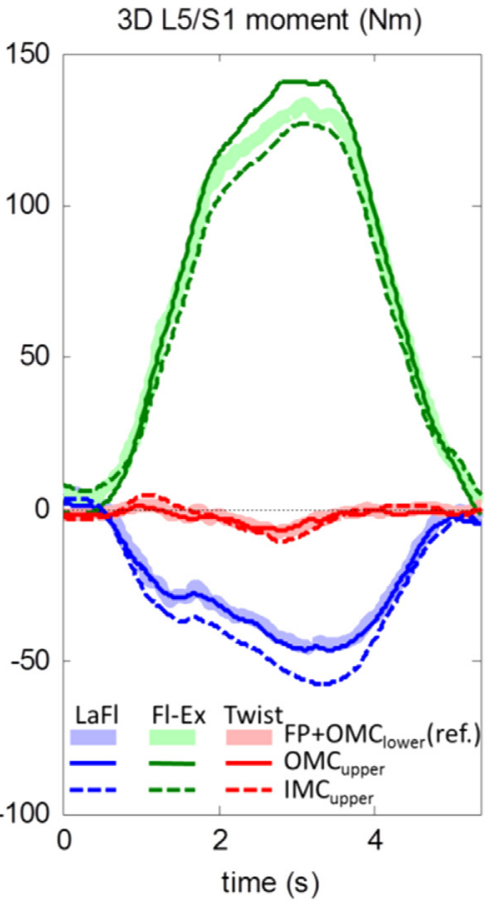

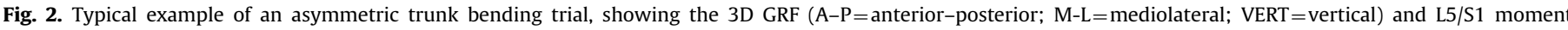

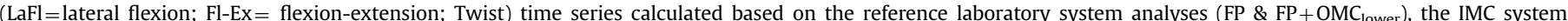
analyses $\left(\mathrm{IMC}_{\text {full }} \& \mathrm{IMC}_{\mathrm{upper}}\right)$, and the additional laboratory system analyses $\left(\mathrm{OMC}_{\text {full }} \& \mathrm{OMC}_{\mathrm{upper}}\right)$. 

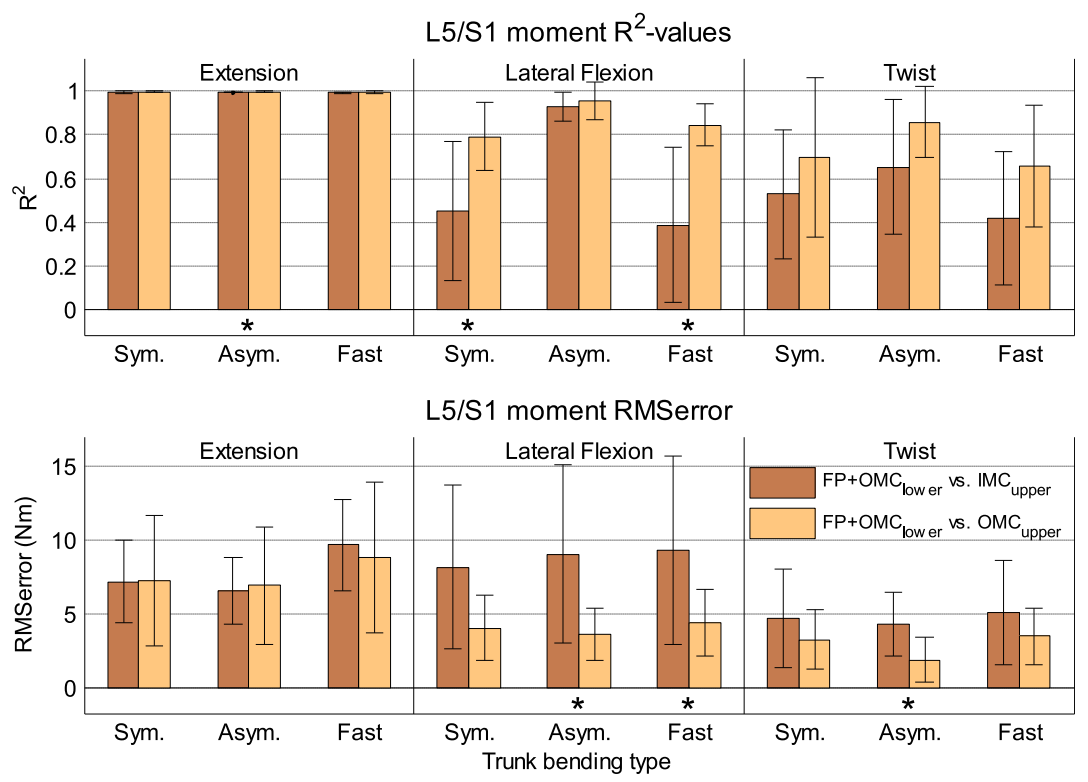

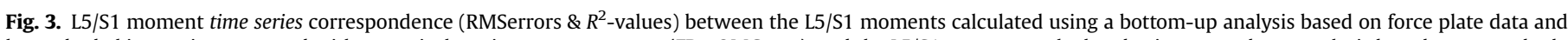

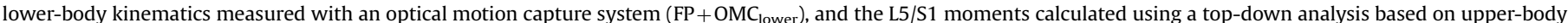

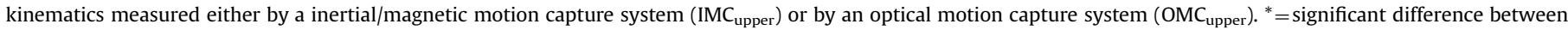

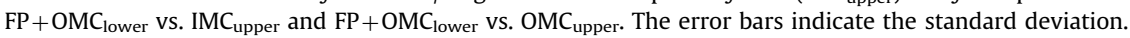

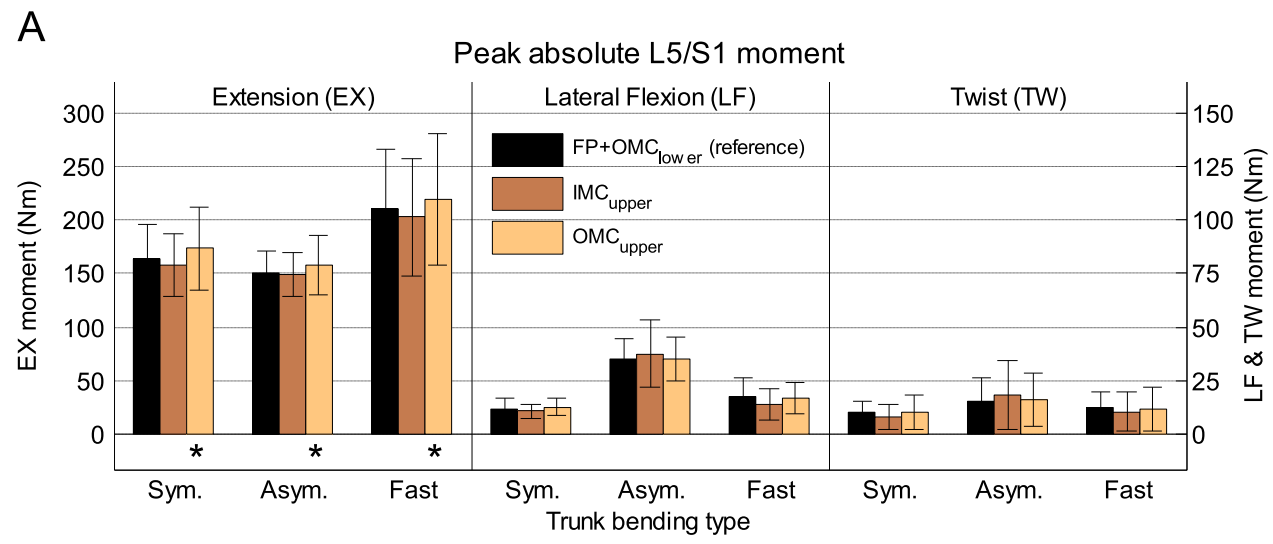

B Peak absolute L5/S1 moment ICC plots
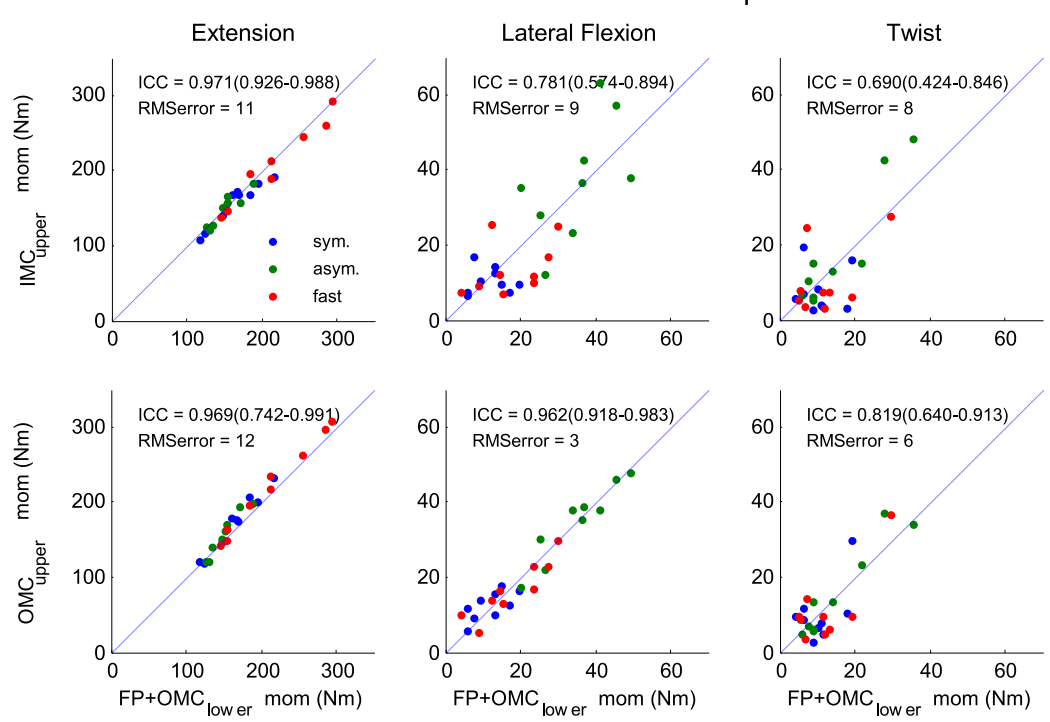

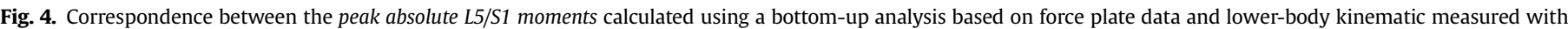

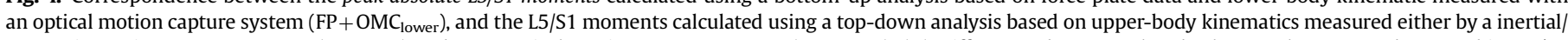

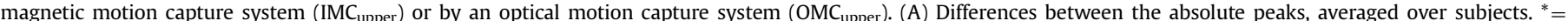

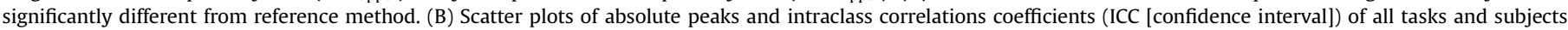
together. Note that non-overlapping confidence intervals indicate a significantly different ICC. 


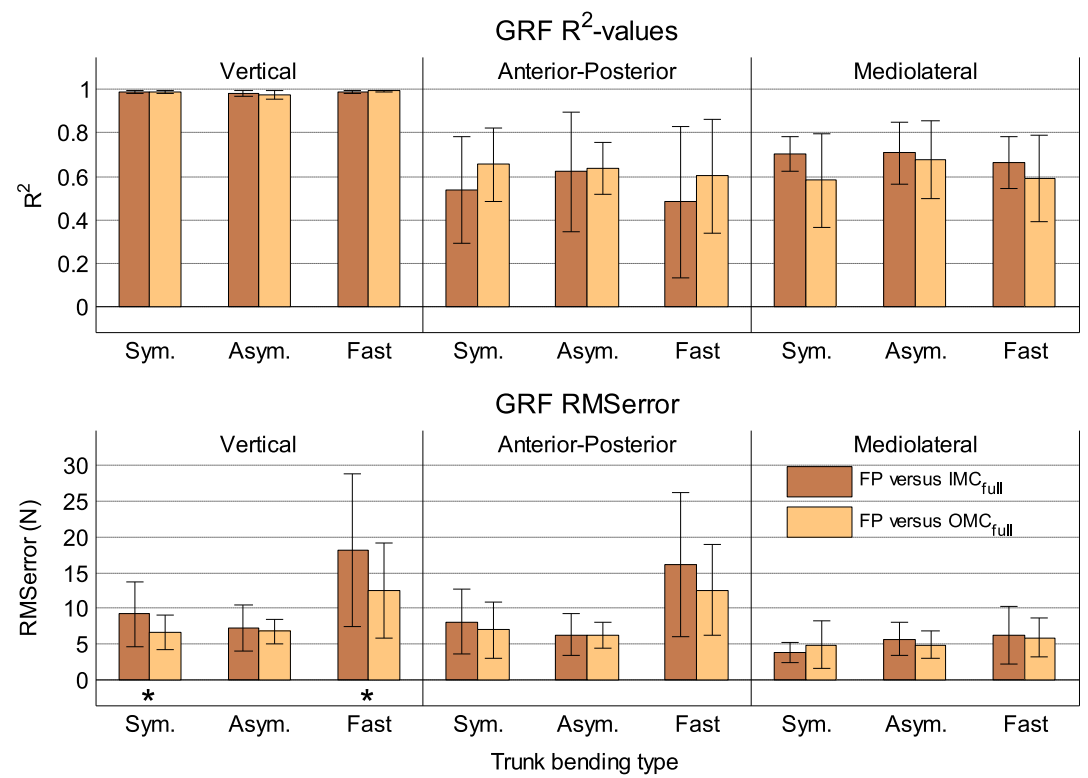

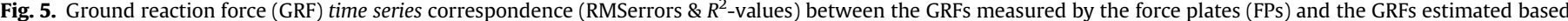

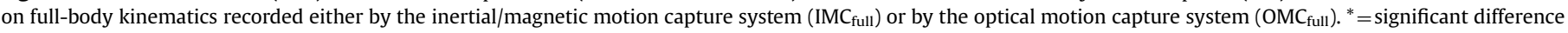
between $\mathrm{FP}$ versus $\mathrm{IMC}_{\text {full }}$ and $\mathrm{FP}$ versus $\mathrm{OMC}_{\text {full. }}$. The error bars indicate the standard deviation.

A

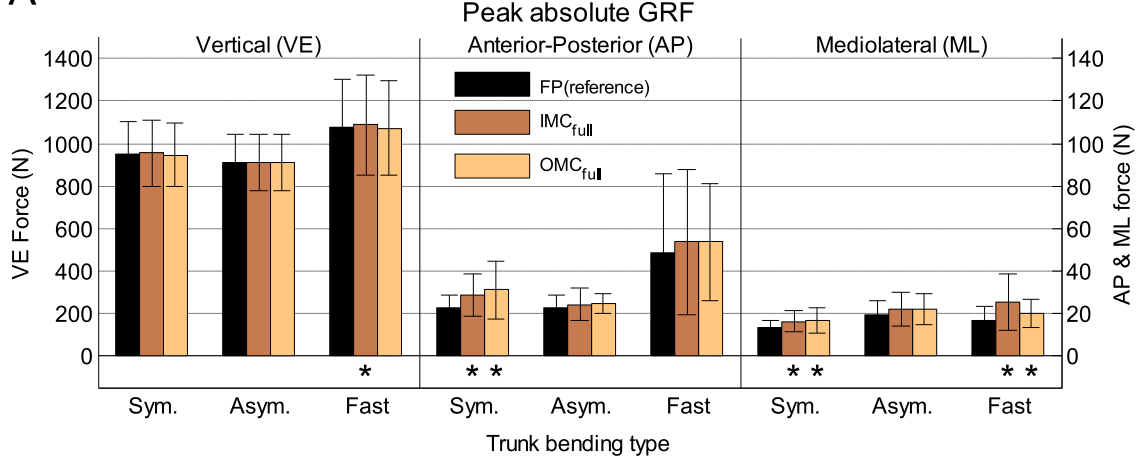

B

Peak absolute GRF ICC plots
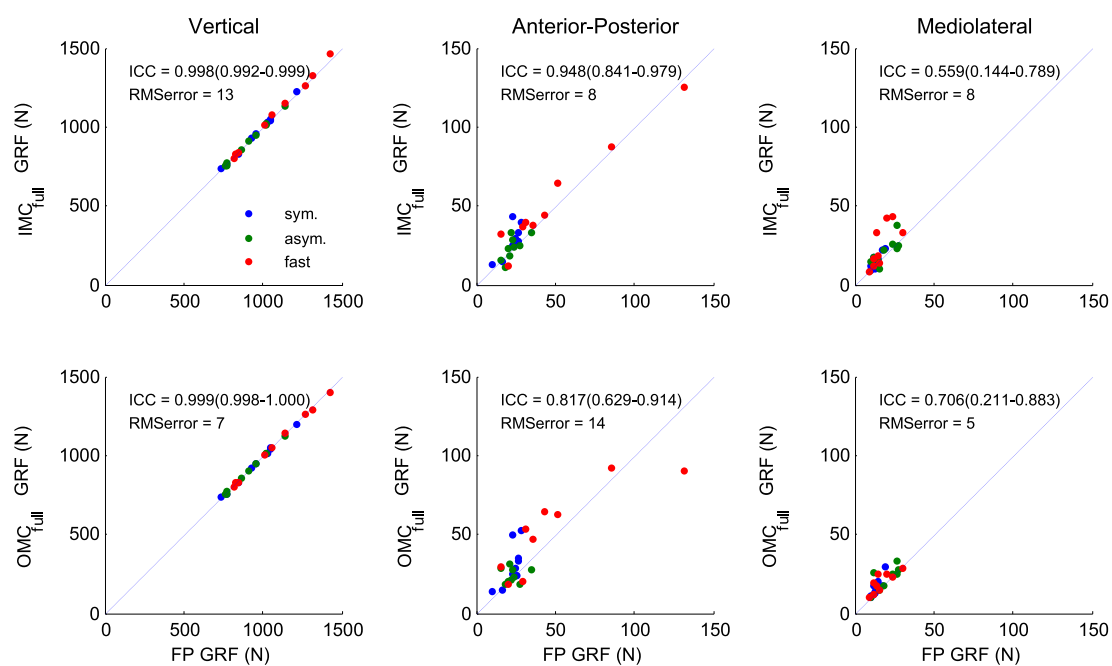

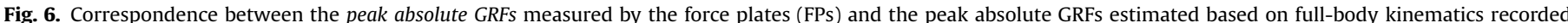

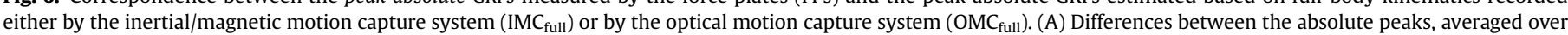

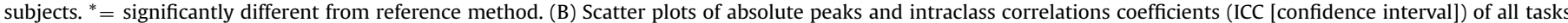
and subjects together. Note that non-overlapping confidence intervals indicate a significantly different ICC. 
estimates from the reference OMC+FP method and the IMC system was very good (Fig. 3), with $R^{2}$-values above 0.993 and RMSerrors below $10 \mathrm{Nm}$ (about 5\% of the peak L5/S1 extension moment).

For the L5/S1 lateral flexion and twist moments, $R^{2}$-values were substantially lower than for the L5/S1 extension moment, especially in symmetric and fast trunk bending (Fig. 3). However, the RMSerrors were not larger (also below $10 \mathrm{Nm}$ ), indicating that the lower correlations were caused by the smaller L5/S1 moment variance rather than less accurate L5/S1 moment estimation by the IMC system.

When applying the top-down inverse dynamics moment estimation method to the OMC data, for the extension and twist moments a similar correspondence with the reference $\mathrm{FP}+$ $\mathrm{OMC}_{\text {lower }}$ method ( $\mathrm{R}^{2}$-values \& RMS errors) was found as when comparing $\mathrm{IMC}_{\mathrm{upper}}$ and reference $\mathrm{FP}+\mathrm{OMC}_{\text {lower }}$ outcomes. For the L5/S1 lateral flexion moment significantly smaller RMSerrors (for symmetric an fast trunk bending) and higher $\mathrm{R}^{2}$-values (for asymmetric and fast trunk bending) were found than in the $\mathrm{IMC}_{\text {upper }}$ versus $\mathrm{FP}+\mathrm{OMC}_{\text {lower }}$ comparison.

\subsubsection{Agreement of L5/S1 moment peaks}

In line with the small RMSerrors, none of the tasks showed significant differences between the reference $\mathrm{FP}+\mathrm{OMC}_{\text {lower }}$ and $\mathrm{IMC}_{\text {upper }}$ peak L5/S1 moments (Fig. 4A). The ICC (Fig. 4B) was high for the L5/S1 extension moment (0.971) and somewhat lower for the L5/S1 lateral flexion (0.781) and L5/S1 twist (0.690) moments.

When using the $O M C_{\text {upper }}$ instead of the $\mathrm{IMC}_{\text {upper }}$ data for the calculation of the top-down L5/S1 moments (the additional calculation), the difference between the top-down and bottom-up average peak L5/S1 moments did not seem to reduce (Fig. 4A), and using $\mathrm{OMC}_{\text {upper }}$ even slightly overestimated peak L5/S1 extension moments. However, judging from the ICC plots (Fig. 4B, lower panel), individual peak L5/S1 lateral flexion moments were more accurately estimated based on the $\mathrm{OMC}_{\text {upper }}$ instead of the $\mathrm{IMC}_{\text {upper }}$ data, resulting in a significantly higher ICC value for the $\mathrm{OMC}_{\text {upper }}$ estimates (0.962 versus 0.781 ).

\subsection{Ground Reaction Forces (GRF)}

\subsubsection{Agreement of GRF time series}

For symmetric and asymmetric trunk bending at normal speed, the correspondence between the vertical GRF measured by the force plate and estimated by the IMC system was good, with $R^{2}$ values above 0.981 and RMSerrors below $10 \mathrm{~N}$ (Fig. 5), which is about $1 \%$ of the peak vertical GRF. For the anterio-posterior and medio-lateral GRF, $R^{2}$-values were substantially lower (around 0.6 ) but the RMSerrors were comparable, suggesting that the lower $R^{2}$ values were related to the smaller GRF variance for those directions rather than less accurate GRF estimation by the IMC system. For fast trunk bending, $R^{2}$-values were comparable to those for the other tasks, while RMSerrors in vertical and AP direction were about twice as high but remained below $20 \mathrm{~N}$ (Fig. 5), which is about $2 \%$ of the peak vertical GRF.

When utilizing the same GRF estimation method, but now based on the laboratory OMC data, similar differences with the FP measured GRF were found in most cases (Fig. 5). Only for the vertical GRF during symmetric and fast trunk bending somewhat smaller RMSerrors were found for the OMC system.

\subsubsection{Agreement of GRF peaks}

In line with the correlations and RMSerrors, the correspondence between the peaks in the vertical GRF (dominant GRF component) measured by the FP and estimated using the IMC system was generally good (Fig. 6A). Only for fast trunk bending, a slight (but significant) systematic overestimation (about $15 \mathrm{~N}$ ) of the peak was found. Furthermore, the ICC (Fig. 6B) of the vertical GRF peaks between $\mathrm{FP}$ and $\mathrm{IMC}_{\text {full }}$ estimation was high (0.998).

The anterior-posterior and mediolateral peak GRF were somewhat overestimated (by about $10 \mathrm{~N}$ ) by the IMC in symmetric and fast trunk bending. In combination with lower peak values and less variance of the peak values over subjects and tasks compared to the vertical GRFs, this resulted in substantially lower ICCs (0.948 for anterior-posterior GRF; 0.559 for mediolateral GRF).

When applying the $\mathrm{IMC}_{\text {full }}$ GRF estimation method to the OMC system data, similar results were found for the mean peak GRF (Fig. 6A), except for the vertical GRF during fast trunk bending, where slightly lower mean peak values were found when using the OMC system (non-significantly different from the reference FP measurements). ICCs were also very comparable and not significantly different from the FP versus $\mathrm{IMC}_{\text {full }}$ ICCs (Fig. 6B, lower panel).

\section{Discussion}

The present study evaluated the performance of a full-body inertial motion capture (IMC) system for estimation of L5/S1 moments and 3D ground reaction forces (GRFs) during trunk bending. As a reference, GRFs were measured with force plates (FPs) and L5/S1 moments were calculated based on data from an optical motion capture (OMC) system and the FPs.

\subsection{Dominant L5/S1 moment and GRFs components}

The results show good correspondence between the IMC and the reference $\mathrm{FP}+\mathrm{OMC}$ measurement systems for the dominant components, the L5/S1 extension moment and the vertical GRFs. Differences between time series were small (L5/S1 extension moment RMSerror $<5 \%$ of peak; vertical GRF RMSerror $<2 \%$ of peak) and the patterns were very similar (L5/S1 extension moment $R^{2}>0.993$; vertical GRF $R^{2}>0.981$;). Furthermore, the peak values in the time series were very comparable (L5/S1 extension moment ICC $>0.971$; vertical GRF ICC $>0.998$ ), with only for fast trunk bending a slight but systematic overestimation of the peak vertical GRF by the $\mathrm{IMC}_{\text {full }}$ (about $15 \mathrm{~N}$ or $1.5 \%$ of the peak vertical GRF). These results support the validity of the dominant components of the IMC-based L5/S1 moments and GRFs.

The performance of the presented IMC-based L5/S1 extension moment estimation seems to somewhat better than the performance reported in previous studies using inertial sensors (Godwin, 2009; Kim and Nussbaum, 2013): RMSerrors < $10 \mathrm{Nm}$ in the current study compared to $15-20 \mathrm{Nm}$ in previous work. However it is unclear if these differences are related to the difference in assessment method (read Sections 4.4 and 4.5 for possible explanations) and/or differences in experimental tasks (e.g. in the study by Kim, the subjects actually lifted a box). We are not aware of any previous papers estimating GRFs based on multiple inertial sensors.

\subsection{Non-dominant L5/S1 moment and GRFs component}

For the non-dominant components, the L5/S1 lateral flexion and twist moments and the anterior-posterior and mediolateral GRFs, the time series differences with respect to the reference measurement system were comparable to or smaller than the differences found for the dominant terms (L5/S1 moment RMSerror $<10 \mathrm{Nm}$; GRF RMSerror $<20 \mathrm{~N}$ ).

Also, the difference in the peak values were comparable to the ones found for dominant components: no systematic differences with the reference measurement system were found for the peak L5/ S1 moments and mean peak GRFs overestimation stayed below $15 \mathrm{~N}$. 
In contrast, in comparison to the results for the dominant components, for the non-dominant L5/S1 moment and GRF components, the $R^{2}$-values of the time series and ICCs of the peaks were much lower. However, considering the comparable absolute errors this is most likely due to smaller signal variance rather than a less accurate measurement.

Previous studies have reported similar performance for the nondominant L5/S1 moments when using inertial sensor systems (Godwin, 2009; Kim and Nussbaum, 2013). Again, because of differences in experimental tasks, making a fair comparison is difficult.

\subsection{Sources of error}

When estimating the GRFs based on the segment accelerations measured by the reference OMC system instead of IMC system, overall results were not much different (only a few small differences for the vertical GRF). This indicates that the errors in the IMC GRF estimations were not mainly a result of sensor measurement errors (e.g. acceleration/orientation) but rather of inaccuracies in the assumptions in the GRF estimation method (e.g. distribution of body mass over segments, center of mass location, non-rigid segments). This implies that future sensor improvements will probably not result in substantially better GRF estimation accuracy.

For the L5/S1 moments, it is important to realize that the reference method has a limited accuracy itself, since the moment is not directly measured but estimated through inverse dynamics. An indication of the accuracy is usually obtained by comparison with a top-down analysis (internal validity), as done in the present study. Thus, if correspondence between L5/S1 moment estimates of the reference laboratory $\mathrm{FP}+\mathrm{OMC}_{\text {lower }}$ system and the $\mathrm{IMC}_{\text {upper }}$ system is comparable to the correspondence between the two lab estimates ( $\mathrm{FP}+\mathrm{OMC}_{\text {lower }}$ vs $\left.\mathrm{OMC}_{\text {upper }}\right)$, this would imply that the accuracy of the ambulatory IMC system is comparable to the accuracy of the lab system.

For the L5/S1 extension moment, this seems to be the case. No significant differences in $\mathrm{R}^{2}$ and RMSerrors were found between $\mathrm{FP}+\mathrm{OMC}_{\text {lower }} \mathrm{vs} \mathrm{IMC}_{\text {upper }}$ and $\mathrm{FP}+\mathrm{OMC}_{\text {lower }}$ vs $\mathrm{OMC}_{\text {upper }}$ time series. Moreover, the $\mathrm{OMC}_{\text {upper }}$ estimates showed even a systematic overestimation with respect to the reference $\mathrm{FP}+\mathrm{OMC}_{\text {lower }}$

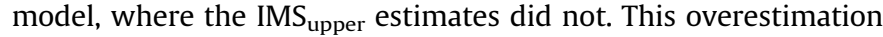
is in line with previous research (Kingma et al., 1996) based on OMC data and might be due to markers on the trunk shifting cranially during trunk bending due to skin motion. This is also a possible explanation for the fact that this problem does not occur when measuring with inertial sensors where the absolute position of the sensors is not measured, but the body is reconstructed based on sensor orientations.

For the asymmetric L5/S1 moment components, the correspondence with $\mathrm{FP}+\mathrm{OMC}_{\text {lower }}$ results was lower for the IMC system than for the $\mathrm{OMC}_{\text {upper }}$ system for the time series $\left(R^{2}\right.$ and RMSerrors) and the peaks (ICCs). This may be related to the errors in capturing angles around the global vertical in the IMC. However, no systematic overestimation or underestimation of the peaks was found. This suggests that the system is suitable for estimating asymmetric L5/S1 moments as long as a sufficient number of participants is measured, to average out these random errors.

\subsection{Alternative data processing}

In the current study, we used the acceleration and angular velocity of the segments that was directly derived from the local IS measurements. Alternatively, segment accelerations could also
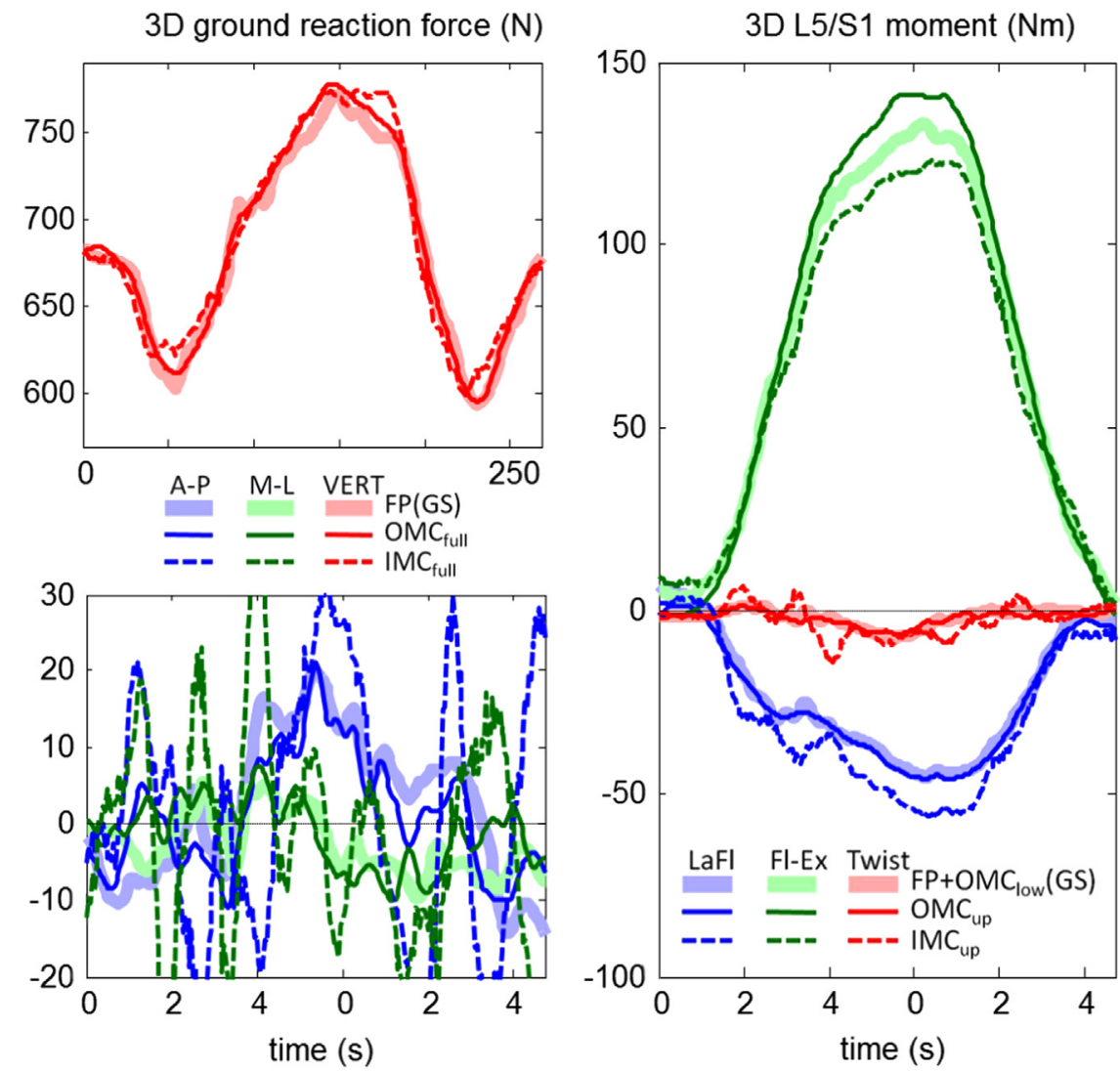

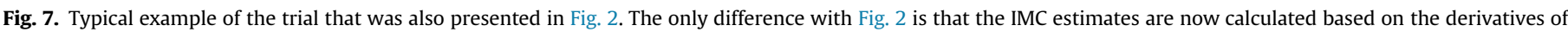

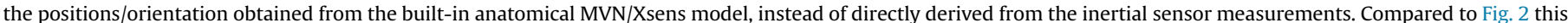
clearly leads to a more noisy result, especially for the Horizontal ground reaction forces. 
have been obtained by taking the second derivative of the position and segment orientations. However, this analysis resulted in more noise, especially for horizontal GRFs (Fig. 7). This is probably because position is not directly calculated based on locally measured accelerations, but the outcome of the built-in MVN bodymodel, consisting of linked body segments. This body model is constructed based on the segment lengths and orientations (which is based on fusion of accelerations, angular velocity and magnetic field signals), and contact points between the feet and floor. All these constraints apparently have a substantial effect on the analyses performed in the present study. Some of the issues mentioned above might also explain the larger L5/S1 moment errors (around $20 \mathrm{Nm}$ ) found in one other study during trunk bending performed by a single subject (Godwin, 2009).

\subsection{Limitations}

It should be mentioned that only healthy male subjects participated in the current experiment. It could be that system performance will be different for women (different anthropometry) and for obese people (mass distribution, wobbling masses). Furthermore, only trunk bending tasks were performed. Future studies should investigate applicability of the pretested estimation methods in other tasks such as walking, running and jumping where impact GRFs may be of special interest (Neugebauer et al., 2014).

Also, the current analysis only provides estimates of GRF and L5/S1 moments due to motion of the body segments. Therefore, in case forces are exerted by the hands (e.g during manual materials handling), the additional GRFs and L5/S1 moments caused by this external force, are not taken into account. Because measuring hand forces is often infeasible in most field studies, hand forces could be estimated by measuring GRF with instrumented force shoes (Faber et al., 2009b) and then subtracting the forces due to body motion as calculated in the present study (Faber et al., 2013a). These estimated hand forces can then be used in a topdown analysis of the L5/S1 moments. It has been speculated (Faber et al., 2013a) that this indirect top-down analysis could perform better than directly using the force shoe GRFs in a bottom-up model because position errors at the end of the segment chain have a larger effect on the L5/S1 moments when the forces are higher (GRF vs Hand forces). This is supported by a few studies using a bottom-up approach based on MVN data (Kim and Nussbaum, 2013) and comparing the use of segment orientation instead of position (Faber et al., 2010), which found larger L5/S1 moment errors than in the current study (errors up to $30 \mathrm{Nm}$ compared to $10 \mathrm{Nm}$ in the current study).

One final limitation is that in the current experiment, subjects started each trial in a homogenous magnetic field, on a platform next to the measurement volume. Thus, the system performance presented here is for a best-case scenario. In the field, system performance might drop due to prolonged magnetic disturbances (Roetenberg et al., 2005). However, as long as the subject keeps moving, the MVN software can make use of its Kinematic Coupling $\left(\mathrm{KiC}^{\mathrm{TM}}\right)$ algorithm, which makes the lower body segments (that are mainly exposed to strong magnetic fields due to metal in the floor) insensitive to magnetic disturbances (Roetenberg et al., 2013). One study (Kim and Nussbaum, 2013) reported that the MVN system has shown a quite robust measurement performance during prolonged measurement in the laboratory, in the vicinity of ferromagnetic objects such force plates and tables with iron frame. More studies are needed to verify this result in the field setting.

\subsection{Conclusion}

This study showed good correspondence between kinetic estimates (L5/S1 moments and ground reaction moments) obtained using an ambulatory inertial motion capture system and a state-of-the-art laboratory measurement system (force plate+optical motion capture system). Thus, the inertial motion capture system is a potential candidate for ambulatory assessment of back loading in field settings. Future work should evaluate the performance of this system during prolonged trials in industrial settings (where the magnetic field is disturbed), and expand the measurement system with measured or estimated hand forces.

\section{Conflict of interest statement}

There are no conflicts of interest.

\section{Acknowledgments}

The first author gratefully acknowledges the Liberty Mutual Harvard T.H. Chan School of Public Health postdoctoral program for supporting this research. The authors also thank Mr. Jacob Banks and Mr. Niall O'Brien at Liberty Mutual Research Institute for Safety for assistance during data collection.

\section{References}

Cappozzo, A., Catani, F., Croce, U.D., Leardini, A., 1995. Position and orientation in space of bones during movement, anatomical frame definition and determination. Clin. Biomech. 10, 171-178.

Coenen, P., Kingma, I., Boot, C.R.L., Twisk, J.W.R., Bongers, P.M., van Dieen, J.H., 2013. Cumulative low back load at work as a risk factor of low back pain: a prospective cohort study. J. Occup. Rehabil. 23, 11-18.

Cutti, A.G., Giovanardi, A., Rocchi, L., Davalli, A., Sacchetti, R., 2008. Ambulatory measurement of shoulder and elbow kinematics through inertial and magnetic sensors. Med. Biol. Eng. Comput. 46, 169-178.

da Costa, B.R., Vieira, E.R., 2010. Risk factors for work-related musculoskeletal disorders: a systematic review of recent longitudinal studies. Am. J. Ind. Med. 53, 285-323.

Faber, G.S., Chang, C.C., Kingma, I., Dennerlein, J.T., 2013a. Estimating dynamic external hand forces during manual materials handling based on ground reaction forces and body segment accelerations. J. Biomech. 46, 2736-2740.

Faber, G.S., Chang, C.C., Rizun, P., Dennerlein, J.T., 2013b. A novel method for assessing the 3-D orientation accuracy of inertial/magnetic sensors. J. Biomech. 46, 2745-2751.

Faber, G.S., Kingma, I., Kuijer, P.P., van der Molen, H.F., Hoozemans, M.J., FringsDresen, M.H., van Dieen, J.H., 2009a. Working height, block mass and one- vs. two-handed block handling: the contribution to low back and shoulder loading during masonry work. Ergonomics 52, 1104-1118.

Faber, G.S., Kingma, I., Martin Schepers, H., Veltink, P.H., van Dieen, J.H., 2009b. Determination of joint moments with instrumented force shoes in a variety of tasks. J. Biomech. 43, 2848-2854.

Faber, G.S., Kingma, I., van Dieën, J.H., 2010. Bottom-up estimation of joint moments during manual lifting using orientation sensors instead of position sensors. J. Biomech. 43, 1432-1436.

Freitag, S., Ellegast, R., Dulon, M., Nienhaus, A., 2007. Quantitative measurement of stressful trunk postures in nursing professions. Ann. Occup. Hyg. 51, 385-395.

Godwin, A., 2009. Investigating the Feasibility of New Methods for Analysis and Collection of Human Motion in Field Applications (PhD thesis). Queen's University, Kingston, Ontario, Canada.

Godwin, A., Agnew, M., Stevenson, J., 2009. Accuracy of inertial motion sensors in static, quasistatic, and complex dynamic motion. J. Biomech. Eng. 131, 114501.

Kim, S., Nussbaum, M.A., 2013. Performance evaluation of a wearable inertial motion capture system for capturing physical exposures during manual material handling tasks. Ergonomics 56, 314-326.

Kingma, I., de Looze, M.P., Toussaint, H.M., Klijnsma, H.G., Bruijnen, T.B.M., 1996. Validation of a full body 3-D dynamic linked segment model. Hum. Mov. Sci. 15, $833-860$.

Kuiper, J.I., Burdorf, A., Frings-Dresen, M.H., Kuijer, P.P.F.M., Spreeuwers, D., Lötters, F.J., Miedema, H.S., 2005. Assessing the work-relatedness of nonspecific lowback pain. Scand. J. Work Environ. Health 31, 237-243.

Kuiper, J.I., Burdorf, A., Verbeek, J.H.A.M., Frings-Dresen, M.H.W., van der Beek, A.J., Viikari-Juntura, E.R.A., 1999. Epidemiologic evidence on manual materials 
handling as a risk factor for back disorders: a systematic review. Int. J. Ind. Ergon. 24, 389-404.

Lötters, F., Burdof, A., 2002. Are changes in mechanical exposure and musculoskeletal health good performance indicators for primary interventions? Int. Arch. Occup. Environ. Health 75, 549-561.

Luinge, H.J., Veltink, P.H., 2005. Measuring orientation of human body segments using miniature gyroscopes and accelerometers. Med. Biol. Eng. Comput. 43, 273-282.

Marras, W.S., Lavender, S.A., Ferguson, S.A., Splittstoesser, R.E., Yang, G., Schabo, P., 2010. Instrumentation for measuring dynamic spinal load moment exposures in the workplace. J. Electromyogr. Kinesiol. 20, 1-9.

Neugebauer, J.M., Collins, K.H., Hawkins, D.A., 2014. Ground reaction force estimates from ActiGraph GT3X+Hip accelerations. PLoS One, 9.

Norman, R., Wells, R., Neumann, P., Frank, J., Shannon, H., Kerr, M., 1998. A comparison of peak vs cumulative physical work exposure risk factors for the reporting of low back pain in the automotive industry. Clin. Biomech. 13, 561-573.

Plamondon, A., Delisle, A., Larue, C., Brouillette, D., McFadden, D., Desjardins, P., Lariviere, C., 2007. Evaluation of a hybrid system for three-dimensional measurement of trunk posture in motion. Appl. Ergon. 38, 697-712.
Plamondon, A., Gagnon, M., Desjardins, P., 1996. Validation of two 3-D segment models to calculate the net reaction forces and moments at the L(5)/S-1 joint in lifting. Clin. Biomech. 11, 101-110.

Roetenberg, D., Luinge, H.J., Baten, C.T.M., Veltink, P.H., 2005. Compensation of magnetic disturbances improves inertial and magnetic sensing of human body segment orientation. IEEE Trans. Neural Syst. Rehabil. Eng. 13, 395-405.

Roetenberg, D., Luinge, H.J., Slycke, P., 2013. Xsens MVN: Full 6DOF Human Motion Tracking Using Miniature Inertial Sensors. Xsens Technologies B.V, pp. 1-5.

Shrout, P.E., Fleiss, J.L., 1979. Intraclass correlations-uses in assessing rater reliability. Psychol. Bull. 86, 420-428.

van den Noort, J.J, van der Esch, M., Steultjens, M.P., Dekker, J., Schepers, M.H., Veltink, P.H, Harlaar, J., 2013. Ambulatory measurement of the knee adduction moment in patients with osteoarthritis of the knee. J Biomech. 46 (1), 43-49.

Vos, T., Flaxman, A.D., Naghavi, M., Lozano, R., Michaud, C., Ezzati, M., Shibuya, K. Salomon, J.A., Abdalla, S., Aboyans, V., et al., 2012. Years lived with disability (YLDs) for 1160 sequelae of 289 diseases and injuries 1990-2010: a systematic analysis for the Global Burden of Disease Study 2010. Lancet 380, 2163-2196. 